\title{
Mechanical Modelings of the Brain and Simulation of the Biomechanism of Hydrocephalus*
}

\author{
Yukio TADA**, Ryuichi MATSUMOTO** \\ and Yuriko NISHIMURA**
}

\begin{abstract}
To know the stress and strain distributions in the brain tissue as a solid is very important for understanding the state or biomechanism of hydrocephalus. But it is impossible to measure such mechanical factors directly in the human body. This paper proposes mechanical models of the brain, whose property has not been clarified, and discusses the adequacy of the models. Computer simulations are performed under the condition that the hydrostatic pressure of the cerebral ventricle is high. Elastic models show the principal stress distributions or distributions of other mechanical factors. Elastoplastic models taking large deformation into consideration show the deformation of the brain tissue. Moreover, simulations that include the "mechanical adaptation" of the living tissue are performed in elastoplastic models. It may be supposed from the result that a mechanical adaptation acts to make the stress distribution uniform in the brain and to promote ventricular enlargement.
\end{abstract}

Key Words: Biomechanical Engineering, Biomechanism, Hydrocephalus, FEM, Simulation, Modeling, Mechanical Adaptation

\section{Introduction}

Hydrocephalus is the state in which cerebrospinal fluid has collected excessively in the head; the hydrostatic pressure in the brain increases and the cerebral ventricle is abnormally inflated. It is one of the most serious diseases in neurosurgery. Conventionally, hydrocephalus is discussed in the context of the hydrostatic pressure. The diagnosis of hydrocephalus is made with CT scan images. However, stress distributions in the brain as a solid which is surrounded by cerebrospinal fluid have yet to be clarified. The large deformation in hydrocephalus cannot be explained by the ordinary mechanics that the cerebral ventricle expands as its wall is compressed by the increasing hydrostatic pressure within. It is considered that besides the compressive deformations due to the hydrostatic pressure in the cerebral ventricle, certain phenomena, such as adaptation, occur.

This paper considers the cause of the cerebral

- Received 6 th October, 1989. Paper No. 88-0339 A

* Faculty of Engineering, Kobe University, Rokkodai, Nada, Kobe, 657, Japan inflation in hydrocephalus by adopting some mechanical models for the brain tissue and simulating the mechanical phenomena with the finite- element method, assuming the states in which hydrocephalus may occur. Moreover, by extracting the abnormal states of certain mechanical factors in abnormal loading conditions, we incorporate the mechanical adaptation characteristic into the model, in which the material coefficients vary by abnormal mechanical factors due to the abnormal hydrostatic pressure.

\section{Hydrocephalus}

The brain tissue is the most important organization in the human body and is guarded by the hard skull. In the central part of the brain, there are four vacancies of complex shapes called the cerebral ventricles. The brain is said to be floating in a liquid called cerebrospinal fluid. Therefore, the brain is considered to be surrounded by liquid. In the normal state, the cerebrospinal fluid circulates in the brain, that is, it is produced in the lateral ventricle, passes through the other ventricles and the spinal cord to the brain surface and is absorbed there. If this circulation is interrupted, or an abnormal production or a hin- 
drance to the absorption occurs, too much cerebrospinal fluid is stored, the pressure in the brain increases and the ventricle becomes inflated. This is the disease known as hydrocephalus. Hydrocephalus is diagnosed by CT scan images ${ }^{(1)}$. Figure 1 shows a set of CT images demonstrating the enlargement of the cerebral ventricle. The cause of this abnormal inflation has not yet been clarified.

Conventionally, the pressure throughout the brain is considered uniform and the hydrostatic pressure of the cerebrospinal fluid is regarded as representative because it can be measured with relative ease. Recently, it has been noted how nonuniformity in the hydrostatic pressure, depending on the region and the pressure difference in the brain tissue, influences the disease situation ${ }^{(2)}$. It is impossible, however, to measure these mechanical factors directly in vivo. Thus, this study aims to investigate the distribution of these unknown mechanical factors by use of the finite-element method (FEM).

\section{Simulation of Hydrocephalus}

In this paper, it is assumed that hydrocephalus breaks out when the pressure of the cerebrospinal fluid in the cerebral ventricle becomes higher than that on the outer surface of the brain; based on this boundary condition, FEM analysis is carried out in order to study the distribution of various mechanical factors and brain deformations. By comparing these factors in a hydrocephalic brain with those in a normal brain where the inner and outer surface pressures are the same, the causes of the ventricle inflation in hydrocephalus are discussed.

As mechanical models of brains, two simple models are considered: the first is a linear elastic model which assumes infinitesimal deformations; the

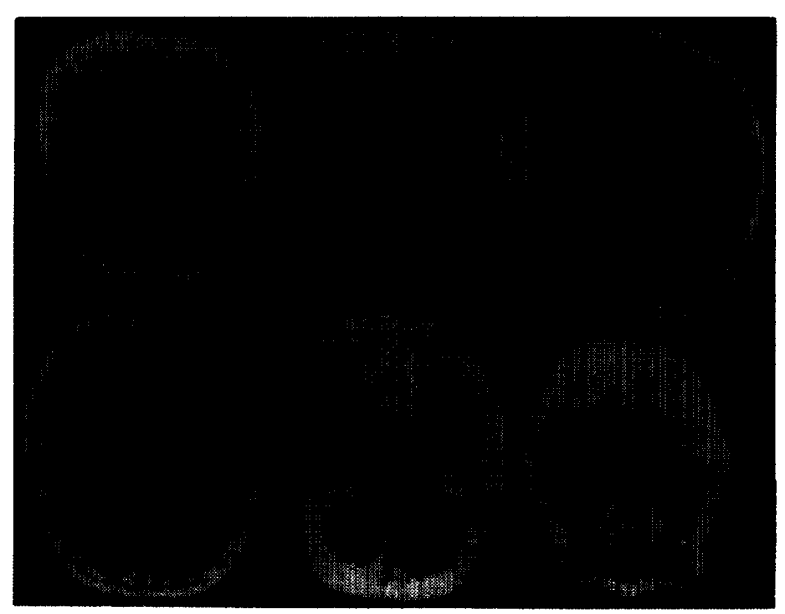

Fig. 1 CT images of a hydrocephalic brain showing the enlargement of the cerebral ventricles second is an elastic-plastic model considering large deformations, which models an irreversible process in the brain tissue.

In both models, the following are assumed:

(1) Though real brains have complicated configurations, the brains are modeled as two-dimensional in the plane strain state and are constructed from CT images of human heads.

(2) The brain models are divided into three parts, that is, brain tissue, cerebrospinal fluid (CSF) and skull. They are all assumed to be homogeneous and isotropic.

(3) The brain configuration and the state of hydrocephalus can be regarded as symmetrical. Thus, only one-half of the model is analyzed. The nodes on the line of symmetry are able to move only on the line, and the bottom node is fixed to prevent rigid displacement.

(4) As for boundary conditions, the pressures of the cerebrospinal fluid in the cerebral ventricle and in the subarachnoid space are both regarded as independent outer compressive loads. We call the former "the internal pressure" and the latter "the external pressure". Both Ioads are applied uniformly on the inner and outer surfaces of the model. In the model of a normal brain, both the internal and external pressures are set at $10 \mathrm{mmHg}$. For the case in which hydrocephalus occurs, the external pressure is $10 \mathrm{mmHg}$, but the internal pressure is set higher than the external, that is, $15 \mathrm{mmHg}$ or $20 \mathrm{mmHg}$.

Figure 2 shows an example of the two-dimensional model analyzed in the FEM simulations.

\section{1 Elastic model}

First, from CT images of a normal brain (its ventricle does not inflate) and from those of a brain in which the cerebral ventricle inflates, two finite-element models are constructed. Second, for both models, simulations with two types of boundary condi-

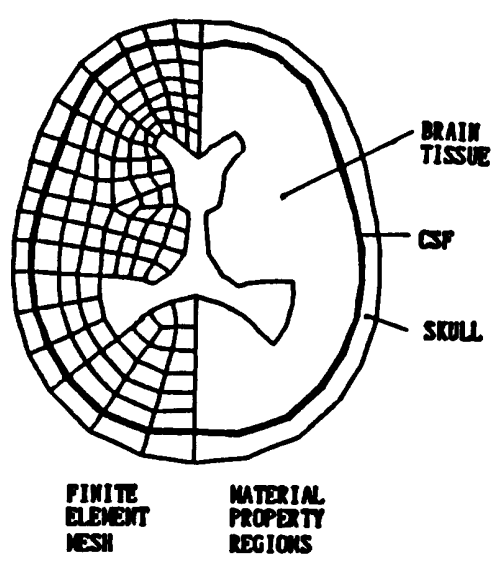

Fig. 2 Two-dimensional finite-element model of a brain 
tions are carried out, one for normal boundary conditions and one for abnormal ones, and the distributions of the principal stresses are compared. The material characters, modulus of elasticity and Poisson's ratio, for brain tissues, cerebrospinal fluid, and skull are not very well-defined or different depending on the earlier works $^{(2) \sim(4)}$. In this simulation, we adopt average values from several papers, as shown in Table 1.

For the normal brain, the distributions of the principal stresses are shown in Fig. 3. In Fig. 3(a), both the internal and external pressures are set to 10 $\mathrm{mmHg}$, and in Fig. 3 (b) the internal pressure is 15 $\mathrm{mmHg}$ and the external pressure is $10 \mathrm{mmHg}$.

Figure 4 shows the results of similar simulations for the inflated brain. In Figs. 3 and 4, and in following figures, the stresses drawn on the left-hand side are the compressive principal stresses and those drawn on the right-hand side are the tensile ones. The stresses in the parts of the skull and cerebrospinal fluid are omitted.

In case (a), where the internal pressure and the external one are both $10 \mathrm{mmHg}$, only compressive stresses appear uniformly throughout the brain irrespective of the brain shape. This result is observed when both pressures are the same, and is not limited to the case of $10 \mathrm{mmHg}$. In case (b), the stress magnitudes are different depending on the elements.

Table 1 Material constants of elastic model

\begin{tabular}{|l|c|c|c|}
\hline & $\begin{array}{l}\text { BRAIN } \\
\text { TISSUE }\end{array}$ & CSF & SKULL \\
\hline $\begin{array}{l}\text { HODULUS OF } \\
\text { ELASTICITY } \\
\left(k g f / c n^{2}\right)\end{array}$ & 0.5 & 0.00000993 & 50000.0 \\
\hline $\begin{array}{l}\text { POISSON'S } \\
\text { RATIO }\end{array}$ & 0.49 & 0.49 & 0.21 \\
\hline
\end{tabular}

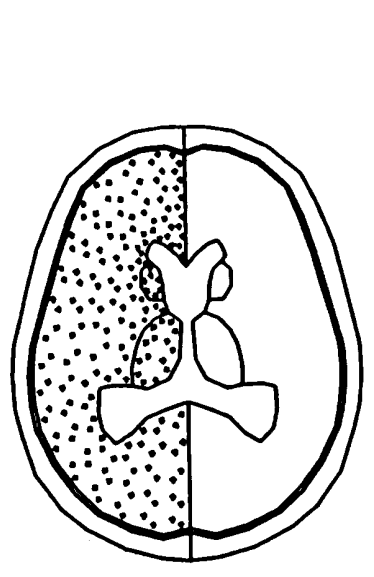

(a)

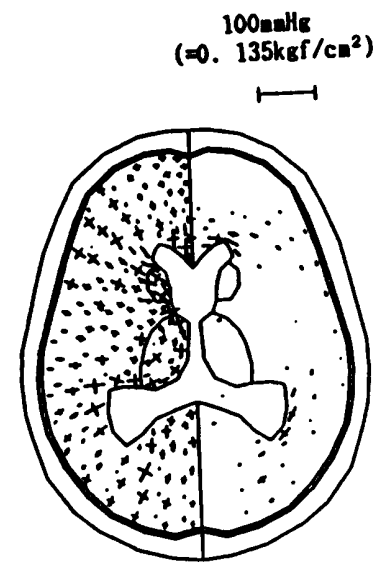

(b)
Fig. 3 Distributions of principal stresses in a normal brain (by elastic model)
In the neighborhood of the anterior and posterior horns of the lateral ventricle, particularly large tensile stresses appear. The area of tensile stress is concentrated close to the wall of the lateral ventricle in the normal brain, but in the inflated brain, that area is widespread.

For the brain models in Figs. 3 and 4, we calculate the distributions of principal strains, principal shear stresses and strain energies as well as the principal stresses, and the areas where remarkable phenomena are observed are limited to the elements near the anterior and posterior horns of the lateral ventricle ${ }^{(3)}$. Therefore, in the following, we discuss the results of simulation by principal stresses.

Next, for three CT images which were obtained from continuous observation of a patient, three finiteelement models are constructed. The CT images were taken at 16th December, 20th December (1985), and 10th January (1986). The boundary conditions of the FEM simulation are that the internal pressure is 10 $\mathrm{mmHg}$ and the external one is $15 \mathrm{mmHg}$. Figure 5 shows the distributions of the principal stresses for these three models. For simplicity, only the results of the brain tissues are drawn.

The tensile stresses appear near the frontal and occipital horns of the lateral ventricle. This area widens in the order (a), ( b ) and (c), and coincides with that of PVL (periventricular lucency).

\section{2 Elastoplastic model}

Generally, brain tissue was treated as viscoelastic ${ }^{(5)}$. Recently, however, it was noted that due to the irreversible nature of the brain, once the cells of the brain tissue degenerate organically, they do not recover. In the following, the brain models are analyzed as elastoplastic in order to incorporate irreversibility into the model. As mentioned before, the

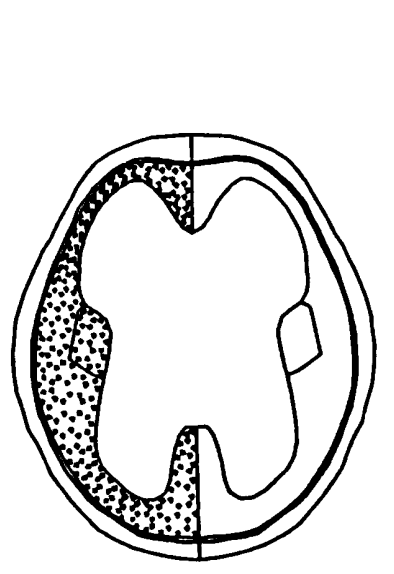

(a)

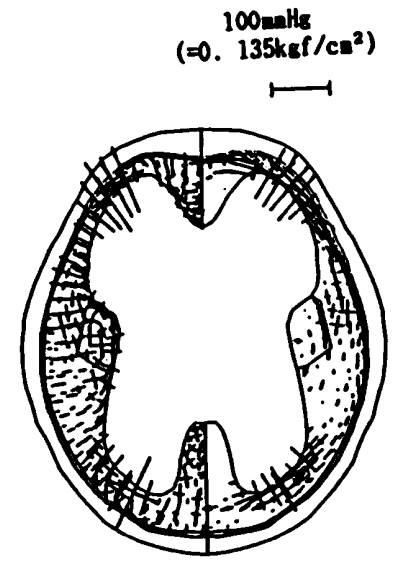

(b)
Fig. 4 Distributions of principal stresses in a hydrocephalic brain (elastic) 


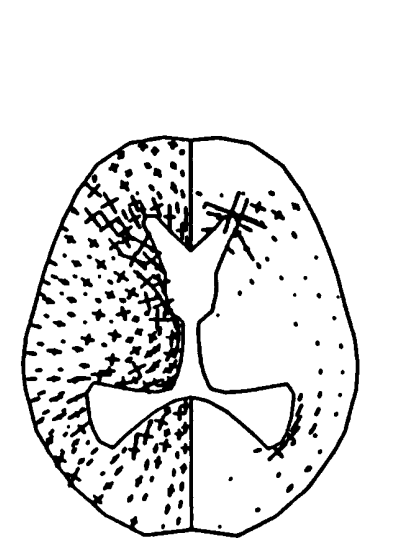

(a) normal state

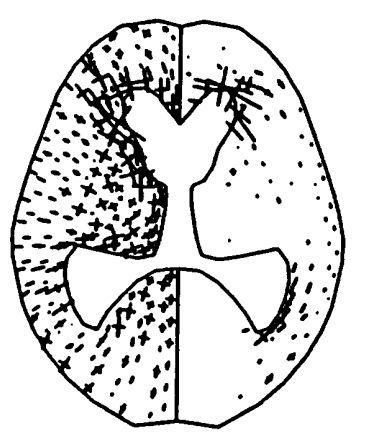

(b) after 4 days

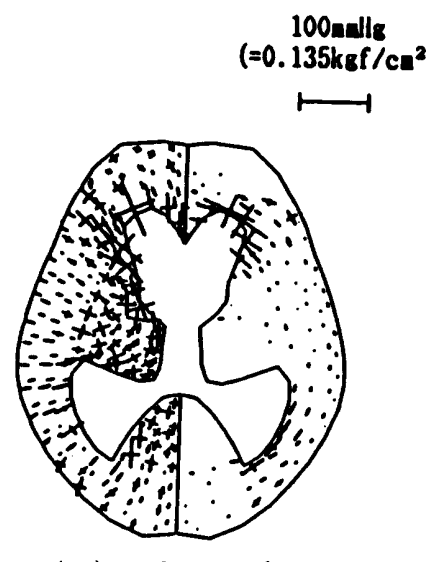

(c) after 25 days

Fig. 5 Stress distributions of models from continuous observation (elastic)

Table 2 Material constants of elastic-plastic model

\begin{tabular}{|c|c|c|c|}
\hline & \begin{tabular}{|l} 
BRAIN \\
TISSUE
\end{tabular} & $\operatorname{CSF}$ & SKULL \\
\hline $\begin{array}{l}\text { MODULUS OF } \\
\text { ELASTICITY } \\
\left(\mathrm{kgf} / \mathrm{cn}^{2}\right)\end{array}$ & 0.12 & 0.00000993 & 50000.0 \\
\hline $\begin{array}{l}\text { POISSON'S } \\
\text { RATIO }\end{array}$ & 0.42 & 0.49 & 0.21 \\
\hline $\begin{array}{l}\text { INITIAL YEILD } \\
\text { STRESS } \\
\left(\mathrm{kgf} / \mathrm{cm}^{2}\right)\end{array}$ & - & 1.0 & 1.0 \\
\hline $\begin{array}{l}\text { HARDEMING } \\
\text { MODULUS }\end{array}$ & 8.0 & 1.0 & 1.0 \\
\hline
\end{tabular}
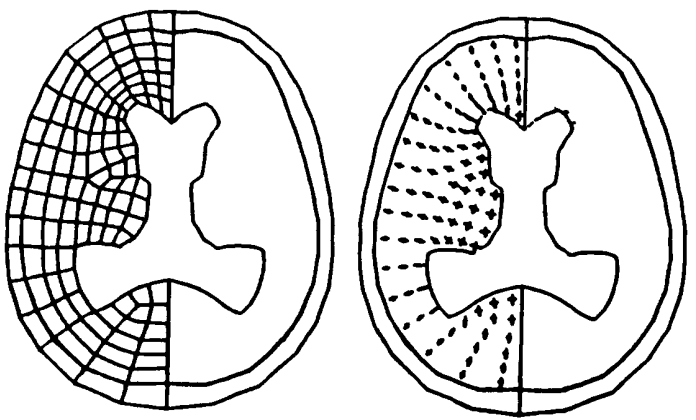

(a) $\sigma_{y}=1.0$

deformations observed in hydrocephalus are very large, so we use the FEM model which takes large deformations into consideration. By this treatment, we can consider not only the stress distributions, but also the deformations. The elastoplasticity of the brain is assumed to be governed by the constitutive equations

$$
\varepsilon= \begin{cases}\sigma / E, & \sigma \leqq \sigma_{y} \\ \left\{\sigma_{y} /(n E)\right\}\left\{\left(\sigma / \sigma_{y}\right)^{n}-1+n\right\}, & \sigma>\sigma_{y}\end{cases}
$$

where $\sigma$ is the true stress, $\varepsilon$ is the natural strain, $E$ is Young's modulus, $n$ is the strain hardening exponent and $\sigma_{y}$ is the initial yielding stress. The constitutive relation for two-dimensional models can be determined by $E, n, \sigma_{y}$ and Poisson's ratio $\nu^{(6)}$. The yielding is judged by von Mises' equivalent stress.

Adopting an initial state from the configuration of the normal brain in which the ventricle is not inflated, FEM simulations are tested by varying the value of the initial yielding stress, $\sigma_{y}$. The other material constants used in the simulations are listed in Table 2. Figure 6 shows the results of simulations for several values of the initial yielding stress; that is, (a) $\sigma_{y}=$ $1.0\left[\mathrm{kgf} / \mathrm{cm}^{2}\right]$, (b ) $\sigma_{y}=0.025$ and (c) $\sigma_{y}=0.015$. In each case, the left-hand side of the brain model in the left figure shows a mesh pattern and the right-hand side shows the region of yielding. The left-hand side

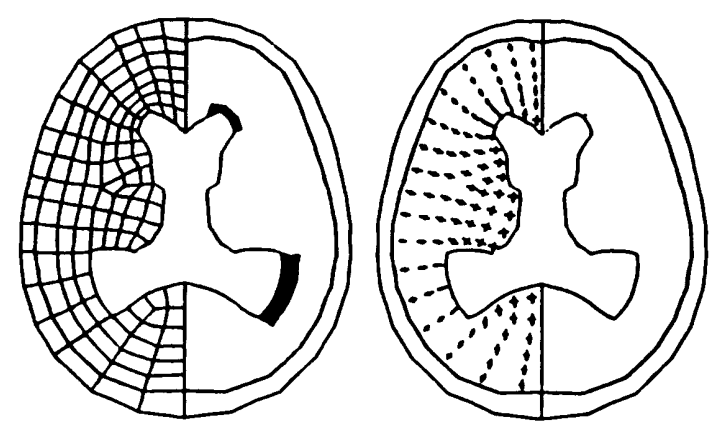

(b) $\sigma_{y}=0.025$

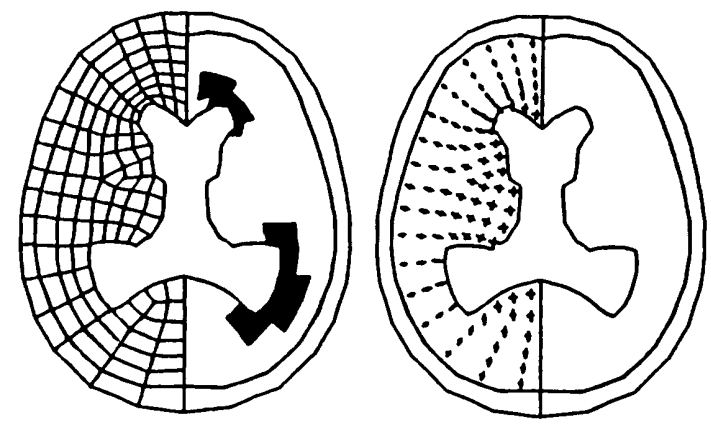

(c) $\sigma_{y}=0.015$

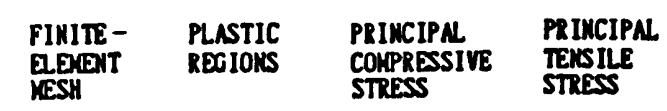

Fig. 6 Yield areas and stress distributions (by elasticplastic model) 
of the right figure shows the compressive principal stresses and the right-hand side shows the tensile ones. In these figures, the stresses in the cerebrospinal fluid and skull are also omitted.

If we assume a high value for the initial yielding stress as in case (a), no plastic region appears. For low values of the initial yielding stress, as in cases (b) and (c), elements near the anterior and posterior horns of the lateral ventricle begin to yield. The deformed states of elastoplastic models resemble the CT image of the hydrocephalic brain shown in Fig. 7 (b) ; there is no noticeable difference between cases (a), (b) and (c) in Fig. 6. Compared to (a) and (b), the deformed shape in case (c) is almost a circular arc, which resembles the actual deformation in the hydrocephalic brain.

We compared deformations by changing the value of the strain hardening exponent, $n$, for a constant $\sigma_{y}$, but the results are almost similar in all cases ${ }^{(3)}$.

The material constants of the brain as an elastoplastic material are unknown at present. From the deformation viewpoint, however, it can be said that the elastoplastic model effectively expresses the deformation of the hydrocephalic brain.

\section{Model Considering Mechanical Adaptation}

Living tissue such as bone has, by its very nature, the abilities of growth and atrophy in that it alters its size and mechanical properties according to its environment, which other engineering materials do not have. This alteration is controlled by stress states as well as by biochemical processes. It is thought that these regulatory actions occur due to anomalies or abnormal changes in the mechanical conditions of that tissue $^{(7)}$. This paper calls these actions " mechanical adaptations" in the sense that it works to restore its mechanical state to normal.

The brain tissue can also be considered to be capable of mechanical adaptation. In this paper, we assume an adaptation model and incorporate it into the FEM simulation models in order to obtain a more realistic model.

The model assumes that first, the living tissue abhors stress concentration on any local area ${ }^{(8)}$. Second, it is observed from CT images of the acute stage of hydrocephalus that tissues around the anterolateral angle of the frontal horn are distorted and destroyed, allowing cerebrospinal fluid to penetrate the brain tissue. This phenomenon is called PVL (periventricular lucency or periventicular low density). With further progress of hydrocephalus, PVL disappears and the shape of the cerebral ventricle, which was complex, becomes spherical. The regions where PVL appears are coincident with the plastic ones in the elastoplastic models.

It can be supposed from these results that the concentration of the tensile stress near the frontal and occipital horns of the lateral ventricle causes the mechanical adaptation. As to the adaptation of living tissues, the configuration type and the material one are possible. This paper considers the latter type, or an adaptation by change of material properties which also reflects the inside stress states. That is, we assume an adaptation model such that if the maximum principal stress of an element exceeds a certain value, the element changes its Young's modulus and

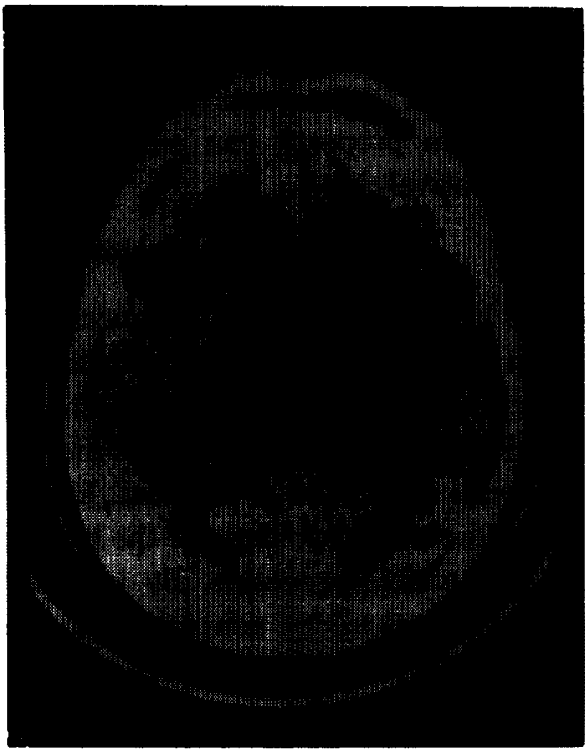

(a) normal state

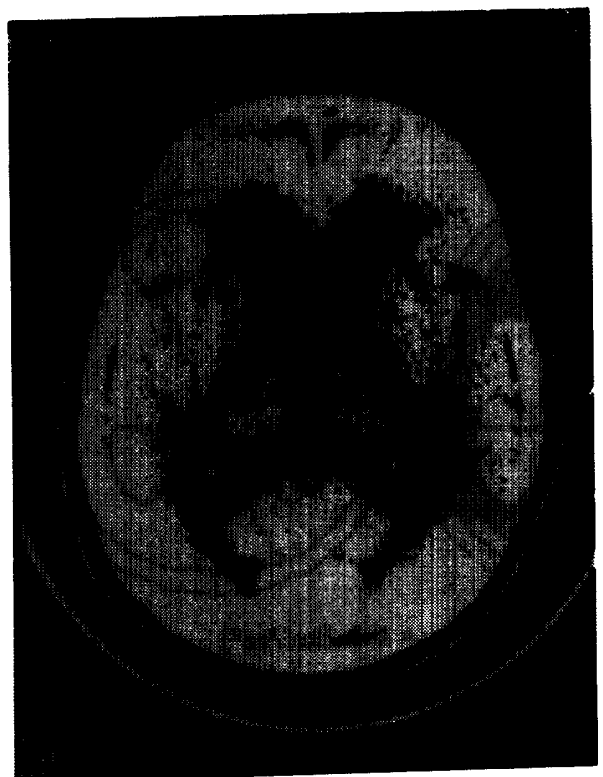

(b) after 25 days

Fig. 7 Examples of CT images 
Poisson's ratio so as to become more flexible. We take the critical stress as the reference value for adaptation and denote it by $\sigma_{a d p}$. More precisely, if the tensile principal stress of each element is beyond $\sigma_{a d p}$, Young's modulus of the element becomes low (from $E$ $=0.5$ to $E=0.05 \quad$ [in $\left.\mathrm{kgf} / \mathrm{cm}^{2}\right]$ ), and Poisson's ratio becomes small (from 0.42 to 0.3 ). Incorporating this adaptation property into the elastoplastic model, the results of the simulations are compared with real CT images of a hydrocephalic brain.

Figure 7 shows CT images of brains: (a) is the

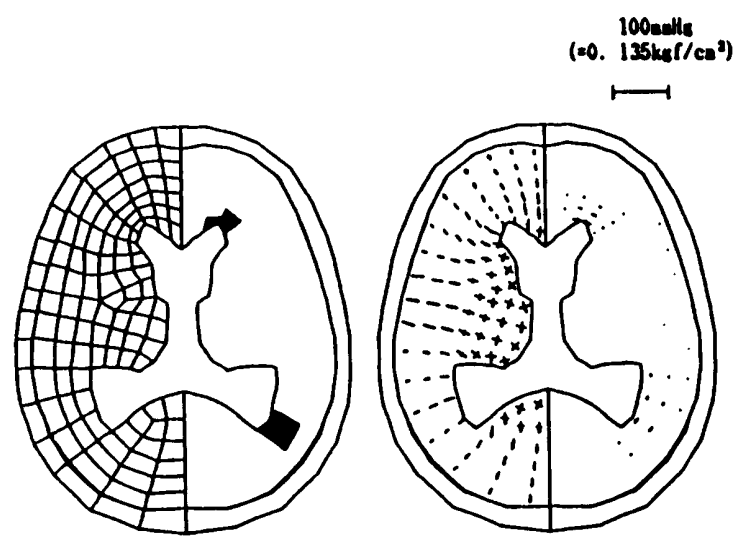

(a) $\sigma_{\text {... }}=0.0135\left[\mathrm{kgf} / \mathrm{cm}^{2}\right]$

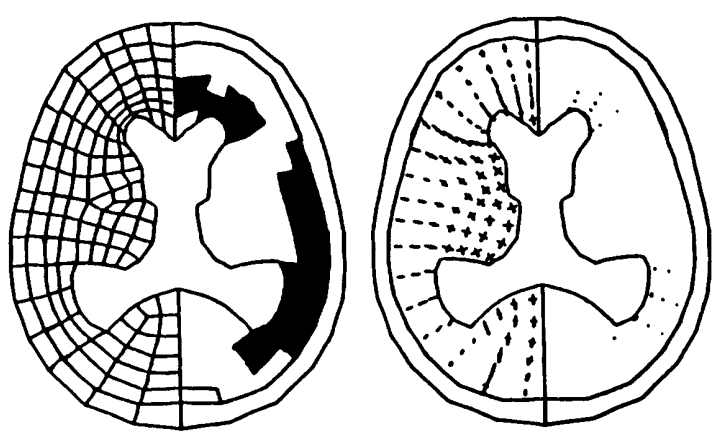

(b) $\sigma_{\text {.co }}=0.0020\left[\mathrm{kef} / \mathrm{ca}^{2}\right]$
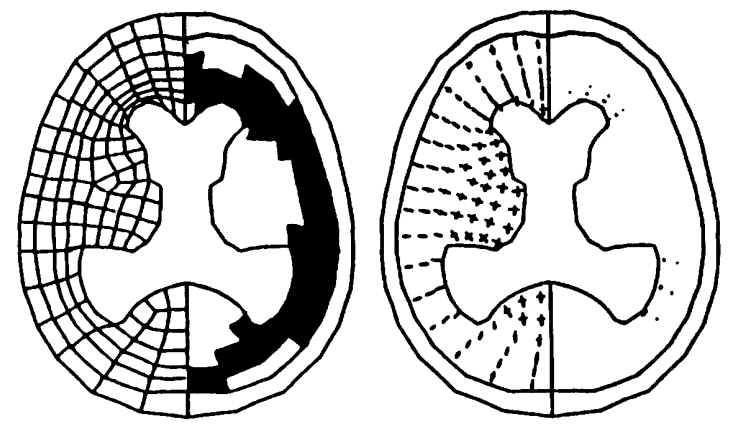

(c) $\sigma_{\text {o.p }}=0.0\left[\mathrm{kgz} / \mathrm{cm}^{2}\right]$

$\begin{array}{llll}\text { PINITE- } & \text { MECHANICN } & \text { PRIHCIPAL } & \text { PRINCIPAL } \\ \text { ELEMENT } & \text { ADAPTATION } & \text { COHPRESSIVE } & \text { TEMSILE } \\ \text { HESH } & \text { REGIOHS } & \text { STRESS } & \text { STRESS }\end{array}$

Fig. 8 Adaptation areas and stress distributions (by elastic-plastic model) brain with no inflation; and ( $b$ ) is the inflated one. Photograph 7(a) corresponds to the model in Fig. 5 (a) and photograph 7 (b) corresponds to that in Fig. 5(c).

The reference values for adaptation are $\sigma_{a d p}=$ $0.0135\left[\mathrm{kgf} / \mathrm{cm}^{2}\right]$ in $(\mathrm{a}), \sigma_{a d p}=0.0020$ in $(\mathrm{b})$, and $\sigma_{a d p}=0.0$ in (c). Initial Young's modulus of the brain tissue, $E$, is $0.5\left[\mathrm{kgf} / \mathrm{cm}^{2}\right]$ and the initial yielding stress, $\sigma_{y}$, is $1.0\left[\mathrm{kgf} / \mathrm{cm}^{2}\right]$. The other material constants are the same as those in Table 2. Figure 8 shows examples of simulation results. In the righthand side of the left figures, elements in which mechanical adaptation occurred are colored black. The figures on the right show the distribution of the principal stresses, as in previous figures.

The mechanical adaptation occurs at the regions between the frontal and the occipital horns of the lateral ventricle. The deformations are largest in case (c), in which $\sigma_{\text {adp }}$ is smallest. comparing the result of Fig. 8(c) with that of Fig. 6(a) which is a simple elastoplastic model and in which the adaptation is not included, the deformations of the central part of the brain are almost the same in both cases, but those of parts around the horns of the ventricle are larger in Fig. 8(c) than in Fig. 6(a). Moreover, in Fig. 8( $\mathrm{c}$ ), which takes the adaptation into consideration, the frontal horn deforms into a circular arc, which more closely resembles the real deformed state of hydrocephalus in Fig. 7 ( b ). It is considered from these results that the introduction of the adaptation property can produce a simulation model closer to real deformation states.

For the present, the mechanical adaptation of the brain is hypothetical, and we cannot make conclusive remarks as to how and why such adaptation occurs. The present model in this paper has no experimental base, but it is interesting that the model with the adaptation property can simulate the deformation comparatively well.

\section{Conclusions}

This paper analyzed the mechanical behavior of brain tissue by modeling it with linear elasticity and with elastoplasticity. Through the analysis of the elastic model, the distribution of mechanical factors in the brain as a solid is clarified. Elastoplastic models can express deformations of the brain tissue including its irreversible processes. By incorporating the concept of mechanical adaptation in the elastoplastic models, the possibility of more realistic simulations is demonstrated in the context of the deformation.

If the mechanical adaptation is ascertained and a mechanical model which can represent the exact mechanism of the brain is constructed, the useful 
application of the simulation grows and we can obtain greater knowledge about the mechanical behavior of living tissue in various kinds of disease, which would be useful in planning medical treatment and surgical operations. Moreover, the mechanism may be utilized for the development of engineering materials.

\section{Acknowledgements}

We are grateful to Prof. Tamaki and Dr. Nagashima, Department of Neurosurgery, Kobe University School of Medicine for their helpful advice.

This research was supported by Grant-in-Aid for Scientific Research 61750083 to Y. Tada from the Ministry of Education, Science and Culture of Japan.

\section{References}

(1) Mori, K. and Handa, H., Clinics for Congenital Anomalies and CT (in Japanese), (1974), p. 25, Nyuron-sha.

(2) Nagashima, T., Tamaki, N., Matsumoto, S. and Horwitz, B., Biomechanics of Hydrocephalus : A New Theoretical Model, Neurosurgery, Vol. 21,
No. 6 (1987), p. 898.

(3) Nishimura, Y., Mechanical Modeling of Brain and Analysis for Biomechanics of Hydrocephalus, Master Thesis of Graduate School of Kobe University, (in Japanese), (1988).

(4) Hosey, R. R. and Liu, Y. K., A Homeomorphic Finite-Element Model of Impact Head and Neck Injury, Proc. Int. Conf. on Finite Element Biomech. (Tucson, Arizona), Ed. by Simon, B. R., Vol. 2 (1980), p. 851.

(5) Tada, Y., Matsumoto, R. and Yamada, H., Viscoelastic Analysis for Biomechanics of Brain by Finite Element Method, Proc. of Jpn. Soc. Mech. Eng. Conf., (in Japanese), No.874-1 (1987), p. 94.

(6) Tomita, Y., Shindo, A. and Kitagawa, H., Bifurcation and Post Bifurcation Behaviour of Internally Pressurized Elastic- Plastic Circular Tubes Under Plane Strain Conditions, Int. J. Mech. Sci., Vol. 23, No. 12 (1981), p. 723.

( 7 ) Fung, Y.C. and Seguchi, Y., Mechanics Applied to Living Systems, Jour. Jpn. Soc. Mech. Eng., (in Japanese), Vol. 88, No. 796 (1985), p. 290.

(8) Fung. Y. C., Biodynamics -Circulation-, (1984), p.54, Springer-Verlag. 